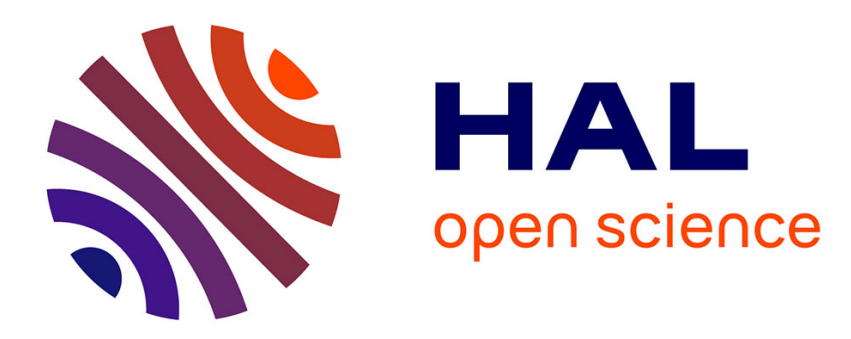

\title{
Modification et caractérisation des surfaces par bombardement ionique
}

E. Knystautas

\section{To cite this version:}

E. Knystautas. Modification et caractérisation des surfaces par bombardement ionique. Journal de Physique IV Proceedings, 1997, 07 (C6), pp.C6-41-C6-53. 10.1051/jp4:1997604 jpa-00255703

\section{HAL Id: jpa-00255703 https://hal.science/jpa-00255703}

Submitted on 1 Jan 1997

HAL is a multi-disciplinary open access archive for the deposit and dissemination of scientific research documents, whether they are published or not. The documents may come from teaching and research institutions in France or abroad, or from public or private research centers.
L'archive ouverte pluridisciplinaire HAL, est destinée au dépôt et à la diffusion de documents scientifiques de niveau recherche, publiés ou non, émanant des établissements d'enseignement et de recherche français ou étrangers, des laboratoires publics ou privés. 


\title{
Modification et caractérisation des surfaces par bombardement ionique
}

\author{
E.J. Knystautas
}

Département de Physique, Université Laval, Québec G1K 7P4

\begin{abstract}
Résumé : Les faisceaux ioniques énergétiques ont servi à doper les semi-conducteurs depuis plusieurs décennies. Moins bien diffusée est l'application de tels faisceaux pour modifier les propriétés tribologiques (microdurêté, usure abrasive, friction) et électrochimiques (corrosion, oxydation) des surfaces métalliques. On peut ainsi former de nouveaux alliages en surface, ainsi que de nouvelles structures, par exemple quasicristallines. Le bombardement ionique peut aussi modifier les propriétés optiques (indice de réfraction, réponse nonlinéaire) des verres et des polymères. Sur un autre plan, les faisceaux ioniques peuvent servir en tant que sondes afin de déterminer les profils de concentration absolue des éléments lourds (par rétrodiffusion Rutherford) ou légers (par détection des ions de recul élastique) dans les premiers micromètres de la surface. Quelques exemples récents de toutes ces applications seront présentés.
\end{abstract}

\begin{abstract}
Although energetic ion beams have been widely used for several decades to dope semiconductors, their application to the modification of the tribological (e.g., microhardness, abrasive wear, friction) and electrochemical (e.g., corrosion and oxidation) properties of metallic surfaces is less widespread. Such ion beams can be used to create new near-surface alloys or new types of structures, such as quasicrystals. The optical properties (such as refractive index or nonlinear response) of materials such as glasses and polymers can also be modified by ion bombardment. On another level, fast ion beams can also be used to probe surfaces by determining the absolute concentration profiles of either heavy elements (by Rutherford Backscattering Spectrometry) or light ones (by Elastic Recoil Detection Analysis). Some examples of these applications will be presented.
\end{abstract}

\section{INTRODUCTION}

Le développerment des accélérateurs pour la physique nucléaire dans la deuxième moitié du vingtième siècle a eu des retombées inattendues dans d'autres domaines de recherche. La ruée vers des énergies de plus en plus hautes, afin de sonder les constituants de plus en plus élémentaires de la matière a laissé un grand nombre de ces accélérateurs (type Cockroft-Walton, Van de Graaff, cyclotron et autres) inutilisés ou sousutilisés. Un certain nombre d'entre eux ont pu se réorienter vers d'autres applications, par exemple, les collisions atomiques, la spectroscopie des ions multichargés, la simulation de dommage par irradiation des parois qui se trouvent dans les réacteurs nucléaires et la préparation d'isotopes radioactifs pour des fins industrielles et médicales. D'autres études ont porté sur les phénomènes reliés à la propagation des atomes énergétiques dans la matière solide, par exemple, le pouvoir,d'arrêt et la canalisation.

Déjà dans les années soixante, l'industrie des semi-conducteurs a réalisé qu'un faisceau d'ions pouvait être un moyen de dopage non-thermique pour les semi-conducteurs intrinsèques. Par conséquent, les accélérateurs ont du être modifiés à cette fin. Au lieu de privilégier une haute résolution en énergie, on a plutôt mis l'accent sur le développement des sources d'ions qui pouvaient produire des faisceaux intenses et souvent, à partir des éléments réfractaires ou corrosifs. Les études sur le pouvoir d'arrêt des ions dans la matière solide, ainsi que sur la canalisation étaient indispensables pour cette application.

Alors que ces implantations dans les semi-conducteurs exigeaient quelques centaines de kilovolts d'accélération, d'autres travaux ont montré que des accélérateurs de quelques $\mathrm{MeV}$ pouvaient servir à l'analyse des surfaces. Une panoplie de techniques ont été créées, entre autres la rétrodiffusion de Rutherford (RBS, pour "Rutherford Backscattering Spectrometry "), la détection des ions de recul élastique (ERDA, pour « Elastic Recoil Detection Analysis »), l'analyse par réactions nucléaires induites par 
le faisceau incident (NRA, «Nuclear Reaction Analysis »), l'analyse par fluorescence $X$ induite par un faisceau de protons (PIXE, " Proton-Induced X-ray Emission »), pour n"en nommer que quelques-unes.

La bibliographie qui traite de toutes ces applications des faisceaux ioniques est beaucoup trop vaste pour être citée ici in extenso, et pour des informations plus complètes, on peut se référer à un certain nombre de volumes, par exemple, les oeuvres de Hirvonen [1], Townsend [2], Agius et al. [3] et Pranevicius [4]:

\section{LA MODIFICATION DES SURFACES PAR IMPLANTATION ET MIXAGE IONIQUES}

\subsection{Historique}

Si la première implantation ionique peut être attribuée à Rutherford en 1906, lorsqu'il a fait pénétrer des ions d'hélium (particules $\alpha$ ) dans une feuille d'aluminium, ce n'est qu'en 1953 qu'on trouve la première thèse de doctorat sur le sujet, par W. G. Cussins à Cambridge. Peu après, W. Shockley (deux fois lauréat du prix Nobel) a obtenu un brevet sur certains aspects du procédé, après avoir réalisé son potentiel pour le dopage des semi-conducteurs.

La technologie de l'implantation ionique pour doper les semi-conducteurs ayant été déjà bien répandue durant les années soixante, il était prévisible que la technique soit appliquée éventuellement à d'autres solides. Parmi les premières applications à des non-semiconducteurs se trouvent celles du groupe de Harwell [5] en Angleterre sur les métaux au début des années septante.

\subsection{Généralités}

Lorsqu'un ion pénètre un solide, deux processus indépendants contribuent à le ralentir. À plus haute énergie, ce sont des électrons de la cible qui, sans changer la trajectoire de l'ion, ralentissent ce dernier par un processus inélastique analogue à la friction. Lorsque l'ion est suffisamment ralenti, il commence à subir des chocs élastiques avec les noyaux de la cible. La trajectoire devient alors une série de parcours aléatoires entre chaque collision, jusqu'à l'arrêt complet de l'ion dans le solide. Le point où ces deux processus ont une influence égale varie selon l'énergie de l'ion incident, avec sa charge nucléaire $Z_{1}$ et la masse $M_{1}$ de ce dernier, ainsi que les valeurs correspondantes $Z_{2}$ et $M_{2}$ des atomes de la cible. De plus, c'est dans la dernière partie de son parcours que l'ion crée le plus de dommage dans le solide, soit directement, soit par effet de cascades, c.-à-d., par une réaction en chaîne créée par le premier choc élastique, qui transfert une partie de l'énergie cinétique du projectile à des centaines voire des milliers d'atomes de la cible. Dans certains cas, ce réarrangement de la structure cristalline (par exemple, d'un semi-conducteur) peut être indésirable, et exiger une recristallisation par recuit subséquente. Dans d'autres cas, comme dans le mixage ionique décrit cidessous, la création d'un grand nombre de lacunes peut aider à obtenir l'effet recherché.

\subsubsection{L'implantation ionique}

Un seul ion de type A ayant une énergie initiale donnée et incident sur une matrice constituée d'atomes de type $B$, sera éventuellement arrêté dans un site soit substitutionnelle soit interstitielle, à une profondeur $R_{p}$. $\mathrm{La}$ distribution finale pour un grand nombre d'ions incidents sera centrée sur $R_{p}$ et aura une épaisseur d'environ $2 \Delta R_{p}$. La proportion relative de $A$ et de $B$ dans cette région implantée est facile à calculer à partir de la dose totale des ions incidents, et de l'épaisseur de la couche implantée. Cette proportion peut avoir une valeur qui dépasse les limites de solubilité de A dans B, car le processus n'est pas thermique. Ainsi on peut former des alliages qui sont loin d'un état d'équilibre. En d'autres termes, l'implantation ionique peut créer des phases homogènes et de composition arbitraire de deux éléments qui normalement ne sont pas miscibles, même à l'état liquide.

\subsubsection{Le mixage ionique}

Pour certaines applications, lorsqu'un élément particulièrement réfractaire (et donc difficile à produire sous forme de faisceau) doit être implanté dans un substrat, ou lorsqu'on doit obtenir une composition uniforme dans toute une région du substrat, il existe une technique complémentaire à l'implantation ionique, soit le mixage ionique. Dans ce dernier, une couche de l'élément à être introduit dans la matrice est d'abord évaporée sur le substrat. Par la suite, l'interface couche évaporée-substrat est inradiée par un faisceau 
ionique (généralement d'un gaz rare). Ce dernier sert à déplacer les atomes de la coùche évaporée vers le substrat et vice-versa, créant ainsi une zone où les deux types d'atome peuvent être mélangés dans les proportions voulues.

Relié au mixage ionique est la technique IBAD (pour « Ion-Beam Assisted Deposition ») [6]. Elle consiste dans le bombardement ionique d'une surface sur laquelle on dépose simultanément un autre élément. Un exemple serait l'arrosage avec un faisceau d'ions d'azote, d'une surface sur laquelle on évapore du titane. Les flux des deux éléments peuvent être contrôlés pour assurer la bonne stcechiométrie. Les deux réagissent durant la déposition et une couche de TiN ayant une bonne adhésion peut être produite à la surface. L'avantage de cette technique est qu'elle permet la formation de couches épaisses,

Les mécanismes responsables pour le mixage ionique sont variés [7], et pour certains aspects encore controversés [8]. Une étude systématique [9] sur les bicouches Ti-acier a montré que le taux de mixage est proportionnel à l'énergie de dommage (c.-à-d. élastique) déposée, au moins pour ce qui est des masses intermédiaires. Chaque mécanisme a son échelle temporelle après le début de l'irradiation qui lui est associée. On attribue l'expression «thermal spike » à la création initiale d'une onde de choc (avec des températures associées dans les dizaines de milliers de degrés et des pressions dans les GPa) durant les premières picosecondes. L'énergie de l'onde est éventuellement thermalisée, et durant la phase balistique un très grand nombre de lacunes sont créées dans la matrice. Celles-ci à leur tour affectent l'interdiffusion subséquente entre les deux types d'atome.

De plus, on a montré récemment [10] l'importance des facteurs chimiques, en particulier la chaleur de mixage $\Delta \mathrm{H}_{\mathrm{m}}$, qui servent à «guider » le processus de diffusion. Des profils Ảuger effectués à l'aide d'un faisceau d'argon de $5 \mathrm{keV}$ démontre que pour les multicouches $\mathrm{Fe}-\mathrm{Al}\left(\Delta \mathrm{H}_{\mathrm{m}}=-0,25 \mathrm{eV}\right)$, il y a interdiffusion à partir d'une température (durant bombardement) d'environ $525 \mathrm{~K}$. Par contre, pour les multicouches Mo$\mathrm{Cr}$, dont l'enthalpie de mixage est de signe opposé $\left(\Delta \mathrm{H}_{\mathrm{m}}=+0,075 \mathrm{eV}\right)$, les pics rétrécissent au fur et à mesure que la température augmente. (Voir figure 1).
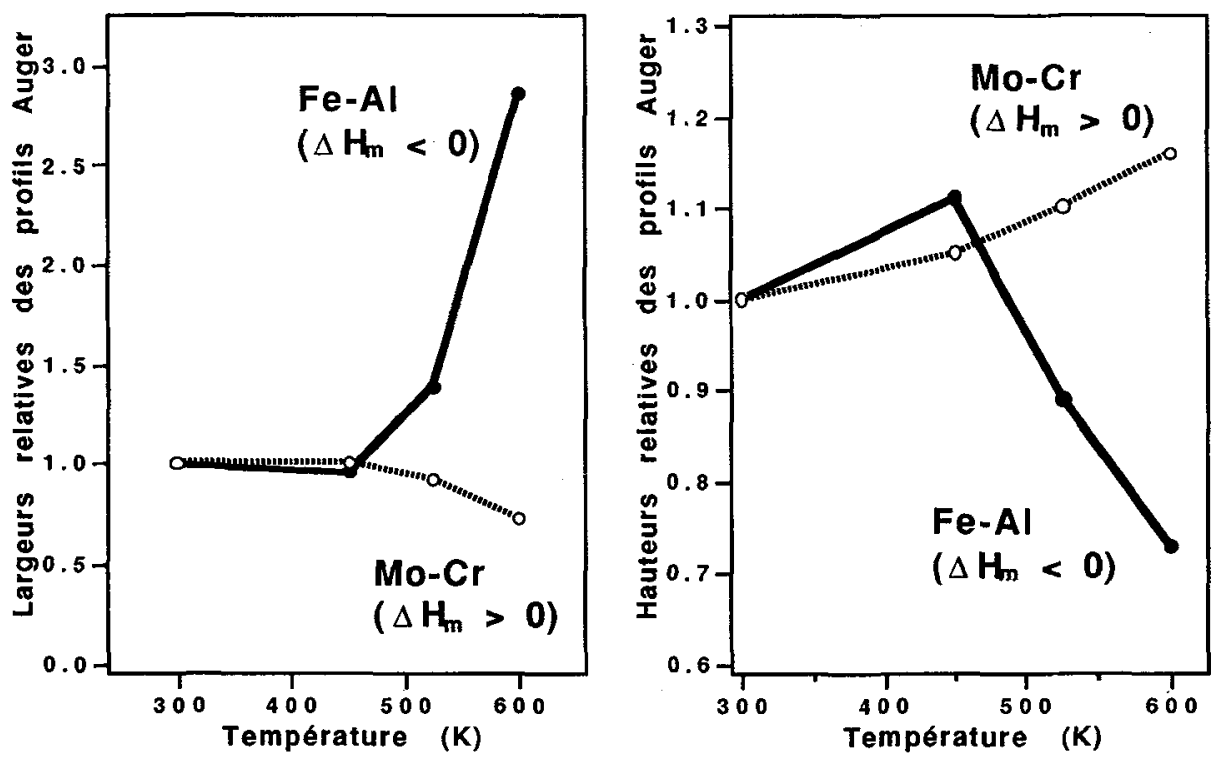

Figure 1. Largeurs et hauteurs relatives des profils Auger pour des multicouches de $\mathrm{Fe}-\mathrm{Al}$ et de $\mathrm{Mo}-\mathrm{Cr}$ en fonction de la tempêrature du substrat. Pour le $\mathrm{Fe}$-Al (chaleur de mixage négative), les pics s'affaissent lorsque la température augmente. L'effet contraire est observé (les pics rétrécissent) pour les multicouches $\mathrm{Mo}-\mathrm{Cr}$ (chaleur de mixage positive). 
Dans les deux cas, la température à laquelle l'interdiffusion augmente peut être associée à celle à laquelle les lacunes commencent à être mobiles. Étant donné qu'une variation de quelques $75 \mathrm{~K}$ du substrat durant le mixage peut avoir un effet aussi marqué, le concept de «thermal spike » semble pour le moins redondant pour expliquer ces comportements.

\subsection{Applications}

L'implantation ionique a déjà été appliquée à plusieurs types de matériaux. Outre les semi-conducteurs, ce sont les métaux qui en ont eu les applications les plus répandues. Ces travaux visaient l'amélioration des propriétés tribologiques (microdureté, usure, friction) et de la résistance à la corrosion et à l'oxydation pour les surfaces métalliques. Une application dramatique a été l'implantation ionique des roulements à billes du moteur principal de la navette spatiale, pour lesquels on a pu réduire l'usure corrosive par deux ordres de grandeur [11].

Étant un processus non thermique, l'implantation peut créer des structures loin de l'équilibre, telles les quasicristaux (voir Sect. 2.3.2 ci-dessous) et les métaux amorphisés. La première démonstration de l'amorphisation de paires métalliques par mixage ionique remonte à 1981 [12]. Dans un travail récent [13] qui compare l'implantation avec le mixage ioniques pour l'amorphisation des métaux purs, on trouve que le mixage peut exiger une dose d'ions plus faible par un orde de grandeur, comparée à l'implantation. Une revue de ce sujet est donné par Rauschenbach [14].

Le fait que l'implantation peut créer une interface avec une gradation de concentrations relatives de deux éléments permet d'accroître l'adhésion entre deux couches, par exemple, l'or et la silice [15]. On peut aussi améliorer l'adhésion des couches "épaisses » (quelques $\mu \mathrm{m}$ ) avec des énergies de quelques $\mathrm{MeV}$ (nécessaire pour pénétrer jusqu'à l'interface), mais même avec quelques centaines de keV quand les ions implantés et le dommage qui leur est associé peuvent migrer au travers des joints de grains jusqu'à l'interface [16].

Dans un moindre degré, l'implantation ionique a été appliquée aux céramiques. Comme dans les métaux, on a constaté une amélioration des propriétés mécaniques dans plusieurs cas [17]. De plus en plus, les céramiques seront utilisées dans les applications à haute température, où la possibilité de remplacer des métaux sera limitée par l'effritement des lubrifiants conventionnels dans de telles conditions. Le mixage ionique offre une solution dans certains cas par la formation d'une couche lubrifiante solide à la surface de la céramique [18].

Un certain nombre de travaux ont également porté sur les polymères. L'implantation ionique peut modifier de façons variées et importantes plusieurs caractéristiques de ces matériaux " faits sur mesure ». On observe un durcissement de la surface et une augmentation importante de la conductivité en surface [19]. D'autres ont noté en plus une réduction du coefficient de friction, de l'usure abrasive, ainsi qu'une augmentation de la résistance à l'attaque chimique et à l'oxydation [20].

Les supraconducteurs à haute température critique peuvent aussi bénéficier de l'irradiation avec des ions énergétiques [21]. Dans ce cas, le bombardement ionique sert à modifier les états d'oxydation dans diverses composantes d'un supraconducteur (le $\mathrm{Bi}-2212)$.

Les applications de l'implantation ionique sont très nombreuses.. À titre d'illustration, on discutera trois exemples d'application tirés de notre laboratoire: la tribologie, les nouvelles structures (quasicristallines) et l'optique nonlinéaire.

\subsubsection{La tribologie des surfaces métalliques}

Depuis un quart de siècle, la formation d'une couche durcie à la surface d'un métal par implantation ionique est devenue monnaie courante en tribologie. La plupart de ces travaux concernent la formation de nitrures des aciers ou des alliages de titane, en vue de les rendre plus résistants à l'usure. Hubler et Smidt [22] résument bien la problématique.

Un exemple récent [23] est la création d'une couche lubrifiante solide (h-BN) à la surface d'un alliage de titane (Ti-6Al-4V) en même temps qu'un durcissement du substrat. Une couche mince de bore (100 $\mathrm{nm})$ est d'abord évaporée sur l'alliage. Par la suite, la surface est irradiée avec des ions d'azote qui pénètrent jusqu'à une distance d'environ $175 \mathrm{~nm}$ de la surface, et donc $75 \mathrm{~nm}$ de profondeur dans l'alliage. (Le plus 
grand dommage est créé en amont du pic de concentration d'azote). La formation de nitrure de titane dans l'alliage a pour effet de durcir ce dernier, alors que l'azote arrêté dans la couche de bore forme le nitrure de bore. Plusieurs méthodes analytiques (profilométrie XPS, spectroscopie infrarouge, Raman et uv-visible) montrent que dans ces conditions, le BN est formé sous forme hexagonale et a la même structure que le graphite. Des mesures tribologiques confirment le durcissement de la surface. La microdurêté Knoop augmente d'environ $50 \%$ pour une charge de $5 \mathrm{~g}$, ce qui se traduit par un durcissement par un facteur de plus que trois pour la couche mince considérée seule. Le coefficient de friction dynamique est réduit, et la résistance à l'usure abrasive augmente (voir figure 2).
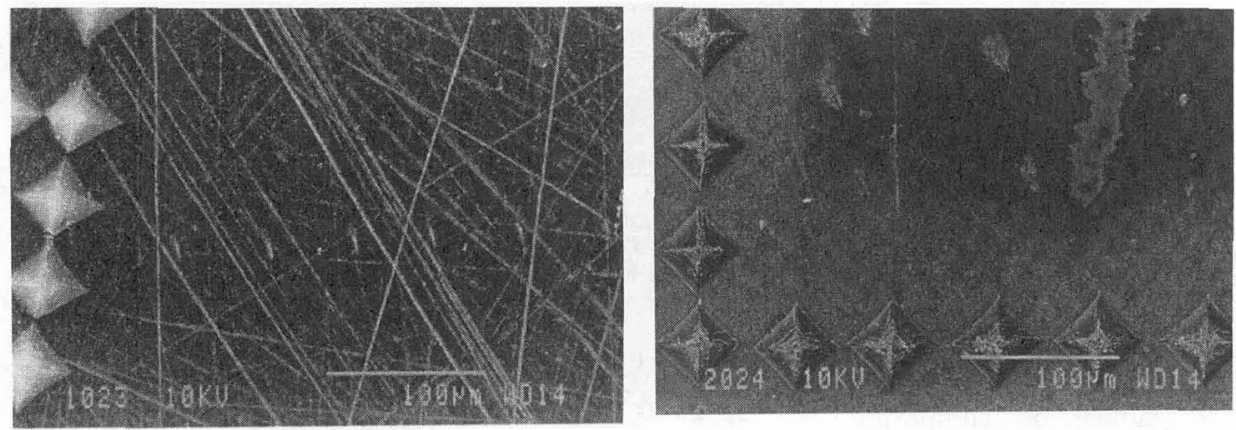

Figure 2. Images de microscope électronique à balayage montrant l'effet de l'usure abrasive sur une surface de Ti-6Al-4V (à gauche) et sur le même alliage traité avec un revêtement de bore ( $100 \mathrm{~nm}$ ) suivi d'une implantation d'azote (à droite). Les indentations Vickers (pour mesurer la microdurêté et l'usure) servent de référence.

\subsubsection{Quasicristaux}

Vers la fin de 1984, lors d'une recherche sur les alliages potentiellement très durs d'aluminium, les premiers quasicristaux ont été découverts [24]. Ces structures ne possèdent pas la périodicité translationnelle des cristaux, elles n'ont pas non plus l'arrangement aléatoire caractéristique des verres et des liquides, mais leurs diagrammes de diffraction aux électrons et aux rayons-X ont des ressemblances à ceux d'un cristal, sauf pour la présence des symétries de rotation (par exemple 5 ou 10) interdites par la cristallographie classique.

Les premiers quasicristaux découverts (et encore les plus étudiés) sont des alliages d'aluminiummanganèse, dans une gamme de composition autour de $\mathrm{Al}_{86} \mathrm{Mn}_{14^{*}}$. Ces structures ont la forme d'un icosaèdre régulier, dont les faces sont formées de vingt triangles équilatéraux. Il s'agit de l'analogue tridimensionnel du pentagone, et fait partie des formes géométriques divines classées comme solides de Platon. D'autres hypothèses ont été avancées pour expliquer les diagrammes de diffraction des quasicristaux, entre autres la présence simultanée de deux structures cristallines " jumellées » (proposée notamment par Pauling [25]) ou par un empilement aléatoire de grandes mailles à symétrie icosaédrique, un type de verre métallique. Mais l'évidence prépondérante semble indiquer que les structures icosaédriques existent dans les faits [26], et même que certaines, dont le $\mathrm{Al}-\mathrm{Li}-\mathrm{Cu}$, maintiennent cette symétrie lorsque chauffées jusqu'à une température près de la fusion.

L'absence de plans de glissement dans les quasicristaux, une conséquence de l'apériodicité translationnelle, suggèrerait des structures très dures en principe, car les dislocations ne peuvent guère s'y propager [27]. Les premiers alliages quasicristallins ont été formés par un refroidissement très rapide (environ $10^{6} \mathrm{~K} / \mathrm{s}$ ) à partir de l'état liquide. Les morceaux ainsi obtenus étaient en fait durs et friables, mais l'inhomogénéité de leur forme rendait leur potentiel d'utilisation en métallurgie plutôt improbable. Par contre, si on pouvait créer de tels alliages à la surface d'une pièce finie, par une tranformation à l'état solide (par exemple, par le mixage ionique d'un revêtement en multicouches des deux - ou trois - composantes élémentaires de l'alliage, et dans la bonne proportion), on pourrait envisager la création d'un revêtement dur (quasicristallin) qui ne changerait pas les dimensions de la pièce à être traitée. Une telle expérience a été réalisée à peine un an après la découverte des quasicristaux [28]. 
À titre d'exemple, la figure 3 montre des diagrammes de diffraction, obtenus grâce à un microscope électronique à transmission, de trois multicouches minces de $\mathrm{Al}-20 \%$ at. Mn traitées par mixage ionique [29]. Avant l'irradiation, on voit des anneaux de diffraction correspondant aux plans cristallins principaux de $\mathrm{Al}$ et de $\mathrm{Mn}$ polycristallins. L'implantation à la température de la pièce aboutit en une structure amorphe. Par contre, à une temperature de mixage de $150^{\circ} \mathrm{C}$, on constate la présence de plusieurs nouveaux anneaux qui correspondent à la structure icosaédrique. (Cette demière peut être considérée comme la projection en trois dimensions d'une structure cristalline sexdimensionnelle, et dans ce schéma d'indexation, six indices sont requis - ils sont indiqués sur la photo.)

Pour une combinaison d'éléments donnée, les quasicristaux sont en général formés dans une gamme de composition restreinte. Parmi les milliers de possibilités d'alliages binaires et ternaires possibles, on peut se demander quelles combinaisons de métaux donneront lieu à des structures

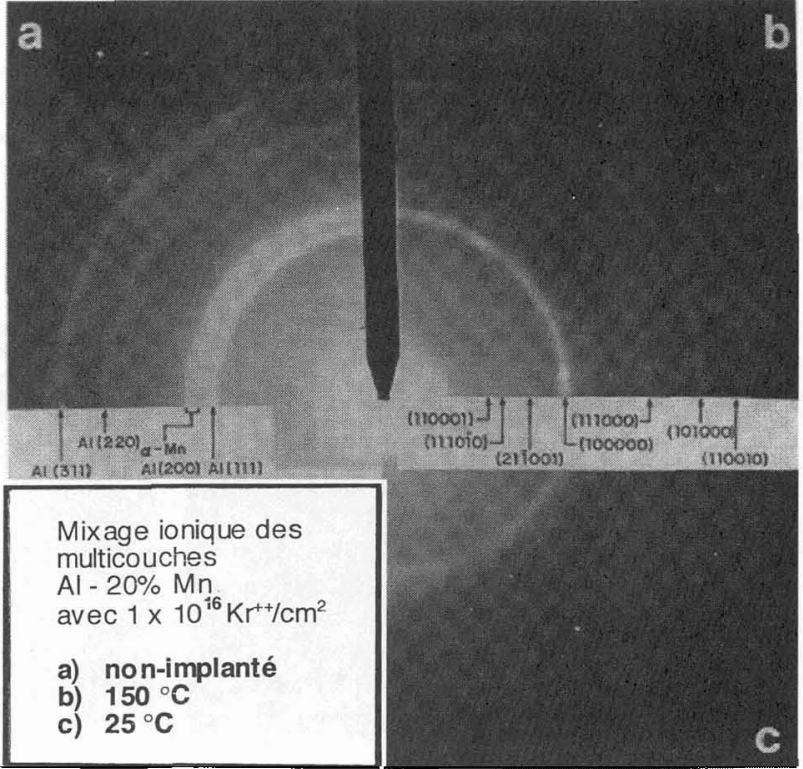

Figure 3. Diagrammes de diffraction des films minces $\mathrm{Al}-20 \%$ at. Mn obtenus par microscopie électronique à transmission: (a) le film tel qu'évaporé, (b) implanté à $150^{\circ} \mathrm{C}$ et (c) implanté à $25^{\circ} \mathrm{C}$

quasicristallines. Â l'aide d'un modèle basé sur les diagrammes structuraux quantiques [30], ayant comme coordonnées une certaine définition d'électronégativité et une définition particulière de rayon atomique, et tenant compte du type d'orbital que les électrons occupent, il a été possible de prédire un sous-ensemble de paires (et groupes de trois) métalliques susceptibles de former des quasicristaux [31]. Deux de ces prédictions ont été testées, aboutissant par la création par mixage ionique des multicouches, de deux nouveaux quasicristaux, un binaire $\left(\mathrm{Ga}_{85} \mathrm{Mn}_{15}\right)$ et un ternaire $\left(\mathrm{Al}_{73} \mathrm{Ni}_{16} \mathrm{Ta}_{11}\right)[32]$.

\subsubsection{Matériaux optiques avancés}

Les possibilités offertes par l'implantation ionique pour la modification des propriétés optiques des semiconducteurs, des cristaux ferroélectriques, des verres et des polymères sont trop nombreuses pour être énumérées ici. Une telle liste occupe une page entière dans un livre récent [33]. Les illustrations qui suivent serviront pour indiquer quelques applications de cette technique.

2.3.3.1 Modification de l'indice de réfraction L'implantation ionique dans les polymères comme le PMMA peut augmenter l'indice de réfraction à la surface [34]. Un dispositif laser permet de suivre l'évolution de l'indice de réfraction et la formation d'un guide durant l'implantation même. Les changements sont attribués à une dépolymérisation et à une compression provenant de la volatilisation des segments polymériques plus courts [35]. De plus, la formation de liaisons doubles $\mathrm{N}=\mathrm{O}$ ajoute à l'augmentation de l'indice. Un exemple récent est la formation de guides optiques dans le $\mathrm{PVCz}$ par implantation d'oxygène [36]. À plus haute énergie (quelques $\mathrm{MeV}$ ), et donc une pénétration plus profonde, il devient possible de former des guides d'onde enfouis.

Dans d'autres matériaux, l'indice de réfraction peut diminuer par quelque pourcent, suite à l'amorphisation d'un cristal (par exemple, le quartz) due au dommage causé par l'ion vers la fin de son parcours [37], ou à la perte des ions de sodium par un verre soda-lime, par exemple [38].

2.3.3.2 Milieux enregistreurs Les films minces de tellurium peuvent servir pour l'enregistrement optique des données. Les qualités recherchées dans de tels matériaux sont un bas seuil pour l'écriture, une bonne résistance chimique et une stabilité à long terme. Dans une étude avec plusieurs ions, on a trouvé que l'implantation de telles couches avec de l'hydrogène, l'hélium, l'azote et l'argon pouvait améliorer leur contraste et diminuer leur seuil d'écriture [39]. La formation de défauts et l'interaction chimique des ions 
avec le tellurium sont associées tous les deux avec les changements observés [40]. L'implantation avec le lithium peut avoir un effet salutaire sur la stabilité de tels films enregistreurs dont le comportement a été suivi sur une période de deux ans [41].

2.3.3.3 Optique nonlinéaire Lorsque la lumière pénètre un solide diélectrique, il y a polarisation des électrons par le champ électrique incident. La polarisation macroscopique $\mathbf{P}$ peut être exprimée en termes d'une expansion du champ électrique $\mathbf{E}$ :

$$
\mathbf{P}=\chi^{(1)}: \mathbf{E}+\chi^{(2)}: \mathbf{E E}+\chi^{(3)}: \mathbf{E E} \mathbf{E}+\ldots
$$

où $\chi^{(1)}$ est la susceptibilité linéaire qui détermine la fonction diélectrique $\varepsilon$, et donc l'indice de réfraction et l'absorption. Les tenseurs de susceptibilité de second $\left(\chi^{(2)}\right)$ et de troisième $\left(\chi^{(3)}\right)$ ordres, qui représentent l'interaction de la lumière avec elle même, sont les plus pertinents pour les applications technologiques de l'optique nonlinéaire.

Il est possible d'augmenter $\chi^{(3)}$ de façon significative par la formation de nanoagrégats métalliques dans une matrice diélectrique, typiquement un verre. Ces agrégats, appelés parfois " quantum dots », ont des propriétés nonlinéaires remarquables à cause de leur faibles dimensions (quelques $\mathrm{nm}$ ). La lumière qui est diffusée par de telles structures métalliques dans une matrice diélectrique a un comportement assujetti aux effets de confinement quantique et qui ne peut pas être adéquatement décrit par un traitement classique.

Il y a plusieurs façons de produire ces nanoagrégats, généralement d'un métal monovalent, dans une matrice de verre. Une des plus directes est par implantation ionique [42]. Plus récemment s'est développée une autre technique, notamment à l'Université de Padoue, qui permet de mieux contrôler la distribution des dimensions des agrégats. Il s'agit de l'échange ionique, soit thermique,soit assisté d'un champ électrique, d'un verre de type soda-lime, suivi d'une irraditaion avec un faisceau d'ions (typiquement $\mathrm{He}$ ou $\mathrm{N}$ ). Lors de l'échange ionique, les ions du métal en solution, typiquement $\mathrm{Ag}$ ou $\mathrm{Cu}$, viennent remplacer les ions de $\mathrm{Na}$ dans le verre. Par la suite, l'irradiation ionique induit une segrégation des ions métalliques introduits par l'échange, en nanoagrégats métalliques. La dose et la température sont deux paramètres qui jouent un rôle qui n'est pas complètement compris dans ce processus. Comme dans tous les cas, l'énergie des ions incidents affecte la profondeur à laquelle les agrégats sont formés.

Les résultats [43] sont montrés aux figures 4 et 5 . La figure 4 montre une photographie des nanoagrégats de cuivre, produits après irradiation avec une dose de $8 \times 10^{16}$ ions $\mathrm{He}^{+} / \mathrm{cm}^{2}$ à $100 \mathrm{keV}$. La figure 5 illustre la réponse optique nonlinéaire d'un échantillon de verre irradié avec $5 \times 10^{16}$ ions $\mathrm{He}^{+} / \mathrm{cm}^{2}$ à $700 \mathrm{keV}$ après échange ionique dans le $\mathrm{CuCl}$. Pour cette mesure par la technique appellée "Z-scan », l'abscisse représente le déplacement de l'échantillon le long de l'axe d'un faisceau laser convergent. Le détecteur a une ouverture ayant un diamètre constant. Lorsque l'échantillon traité passe par $\mathrm{z}=0$ (le point focal du faisceau laser en absence de l'échantillon), son indice de réfraction nonlinéaire (qui varie avec l'intensité) cause une divergence du faisceau pour $z<0$ (et donc un signal plus faible dans le détecteur), et une convergence du faisceau pour $\mathrm{z}>0$, avec une augmentation du signal détecté. D'autres travaux sont en cours pour élucider les mécanismes responsables de la formation des nanoagrégats et de leur effet sur la diffusion de la lumière.

\subsection{Comparaison de l'implantation et du mixage ioniques avec d'autres techniques pour application industrielle}

Les techniques ioniques comportent certains avantages lorsque comparées à d'autres pour le traitement des surfaces. Il s'agit de processus non-thermiques qui sont loin de l'équilibre thermodynamique et donc qui peuvent donner lieu à des alliages impossibles à créer autrement. De plus, il n'y a pas d'interface qui risque d'engendrer des problèmes d'adhésion. Un profil arbitraire des ions implantés est possible, par la simple variation de la dose et de l'énergie des ions. Presque tous les éléments du tableau périodique peuvent être employés, qu'ils soient réfractaires, corrosifs ou radioactifs. Les traitements avec faisceau ionique sont chimiquement propres, contrairement à ceux nécessitant un plasma par exemple, qui contient toujours des impuretés. Finalement, ces traitements peuvent se faire après la dernière étape de finition, car ils ne chargent pas ou peu les dimensions de la pièce traitée. Ceci peut être crucial dans certaines applications comme les outils de précision. 


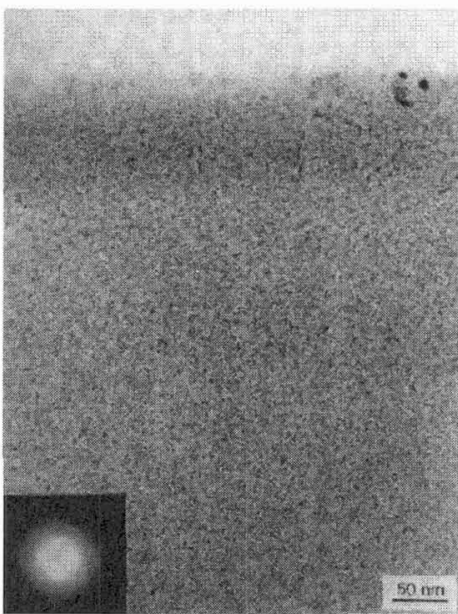

Figure 4. Image (et diagramme de diffraction) par microscopie électronique à transmission d'un verre sodalime traité par echange ionique avec du cuivre, suivi par l'irradiation avec un faisceau d'ions d'hélium à $100 \mathrm{keV}$.

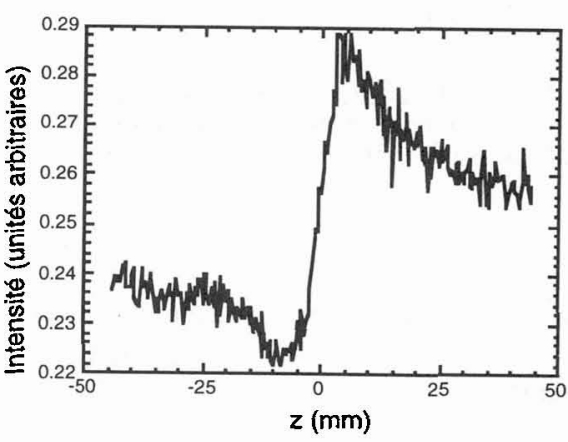

Figure 5. Mesure « Z-scan » d'un verre soda-lime contenant des nano-agrégats de cuivre formés par échange ionique suivi d'irradiation avec des ions d'hélium.

Au chapitre des inconvénients, on peut citer le coût de l'investissement initial, qui doit être amorti sur un nombre relativement restreint de pièces, vu que le tout doit se faire sous vide, et que l'implantation se fait en ligne directe (c.-à-d. qu'une pièce autre que plane doit être tournée lors de l'exposition au faisceau de sorte que toutes ses faces soient irradiées par les ions). C'est pour cette raison que le nombre d'industries qui utilisent couramment les implanteurs, mises à part celles qui font le dopage des semi-conducteurs, est très petit, malgré le quart de siècle qui s'est écoulé depuis les premiers travaux. Des exceptions notables sont les laboratoires de Harwell en Angleterre, et la société américaine Spire Corporation à Boston. Cette dernière se spécialise, entre autres, dans le traitement de prothèses de coudes et genoux, un cas où le coût du traitement est un facteur secondaire.

Face à ces considérations, on est en droit de se demander si l'implantation ionique demeurera pour toujours un outil académique, dédié uniquement à la recherche fondamentale. Des travaux récents suggèrent le contraire, et indiquent que les techniques ioniques ont et auront un rôle à jouer dans le traitement des surfaces qui est complémentaire aux techniques classiques.

La figure 6 compare l'implantation ionique avec la PVD («Physical Vapour Deposition ») et la CVD ( Chemical Vapour Deposition ") soit thermique ou assistée par plasma. On note que l'effet sur les dimensions de la pièce finie crô̂t avec la température du traitement, ce qui avantage l'implantation ionique, un procédé non-thermique, lorsque le facteur dimension est critique pour la pièce finie. On trouve également quelques exemples d'applications pour ces quatre techniques. Récemment on a commenté la difficulté de contrôler la qualité, la reproductibilité et l'adhésion des revêtements formés par PVD et CVD dans l'industrie de l'outillage [44].

Il faut aussi reconnaître que l'investissement capital initial pour un dispositif d'implantation est généralement plus élevé que pour d'autres processus, de type PVD par exemple. Ceci explique pourquoi l'implantation a servi jusqu'ici pour le traitement des pièces ayant une valeur intrinsèque élevée, par exemple dans le domaine médical ou l'industrie des semi-conducteurs. Avant de voir l'implantation ionique plus répandue dans d'autres industries, le coût unitaire de traitement d'une pièce doit décroître de façon importante. Les nouvelles sources d'ions peuvent maintenant fournir des courants des centaines de milliampères voire même des ampères, au lieu des milliampères. De plus, ces faisceaux intenses peuvent être balayés sur des surfaces ayant des dimensions de l'ordre de $100 \mathrm{~cm}^{2}$, au lieu de $10 \mathrm{~cm}^{2}$, qui est courant dans le dopage des semi-conducteurs, les galettes de ces derniers ayant des diamètres typiques de 10 à 15 $\mathrm{cm}$. En somme, la lenteur avec laquelle l'implantation ionique a progressé dans diverses industries est le résultat d'une utilisation inefficace de la technique, et de design qui n'est pas approprié dans un milieu industriel, plutôt que d'un défaut intrinsèque [45]. 


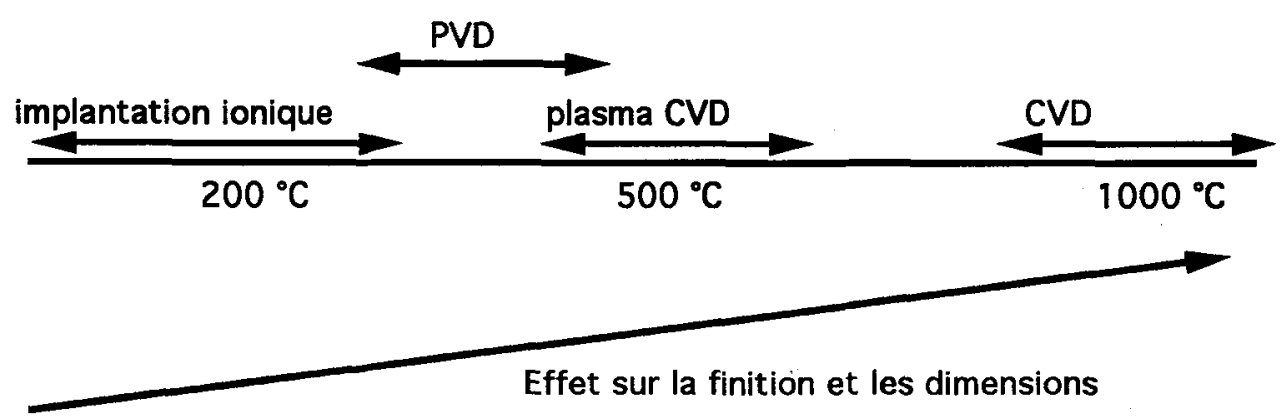

Types d'applications:

Usure adhésive
Usure abrasive (légère)
Corrosion

\section{Exemples d'applications:}

Outils pour couper le caoutchouc, le papier, les plastiques, la viande Outils pour former des lames métalliques minces
Outils de machinerie, forets, poinçons, filières

\begin{abstract}
Sévère usure abrasive
Outils assujettis à des

forces importantes
\end{abstract}

\author{
Usure adhésive \\ Usure abrasive(modérée) \\ Corrosion
}

Outils de machinerie,
Outils pour former
des lames d'inox

Figure 6. Comparaison de quelques techniques de traitements de surfaces, limplantation ionique, PVD et CVD (thermique ou avec plasma).

L'implantation ionique par plasma (PII) a été développée en 1987 comme alternative à l'utilisation d'un faisceau d'ions [46]. Dans cette technique, la pièce à être traitée est placée dans une enceinte où est créé un plasma. Par la suite, une brève impulsion de haute tension négative typiquement de $40 \mathrm{kV}$, est appliquée à la pièce. Les ions positifs du plasma sont accélérés vers la pièce, et la bombardent de toutes les directions. Cette technique traite toute la pièce d'un seul coup ainsi évitant une rotation sur plusieurs axes qui est nécessaire avec un faisceau d'ions conventionnel. Par contre, la dose est plus difficile à contrôler, et il y a une large distribution dans l'énergie (et donc la profondeur) des ions implantés.

Un travail récent [47] compare l'implantation ionique conventionnelle et celle par plasma, avec les techniques standard de nitruration ionique et gazeuse. Lors de traitements d'un acier inoxydable avec de l'azote par les quatre techniques, dans les mêmes conditions de température $\left(400^{\circ} \mathrm{C}\right)$ et de durée $(30$ et 60 minutes), on a trouvé que c'était l'implantation ionique qui a procuré la plus grande concentration et profondeur d'azote dans la matrice, suivie de la PII et des nitrurations. Les mesures tribologiques subséquentes ont confirmé les analyses par profilométrie Auger. On a attribué cet effet au fait que l'énergie plus élevée des ions incidents dans l'implantation classique surmonte mieux les barrières à la surface de l'acier grâce à la pulvérisation cathodique de cette surface, ainsi assurant la pénétration rapide des atomes d'azote vers l'intérieur et leur diffusion subséquente dans la matrice.

Une évaluation réaliste et comparative de l'implantation et du mixage ioniques, ainsi que de la PII, a été effectuée récemment en vue de l'application industrielle de telles techniques [48].

Une application inusitée pour l'implantation ionique servirait pour la catalyse, par l'implantation des poudres. Un projet pilote [49] a pu traiter des quantités de seulement $50 \mathrm{~g}$ à la fois, mais les auteurs suggèrent qu'avec un meilleur design et les nouvelles sources d'ions, il devrait être possible de traiter les poudres catalytiques en continu, ainsi rendant le processus économiquement viable. On signale aussi la possibilité de créer de nouvelles combinaisons porteur/substrat irréalisables par d'autres méthodes et qui auraient des propriétés encore inconnues. 
Un autre processus qui peut créer des alliages loin de l'équilibre est la fusion en surface par faisceau laser. On peut par cette technique traiter des couches plus épaisses. Une étude sur l'oxydation du fer a comparé cette méthode avec l'implantation ionique [50]. Cependant la finition de la surface peut être plus affectée que dans le cas de l'implantation ionique.

Pour clore cette section, on peut citer l'exemple d'un centre technique danois où on a pu développer une niche dans le marché dans laquelle l'implantation est économiquement rentable [51]. Parmi les applications on trouve le traitement des couteaux pour couper le papier, la viande, le caoutchouc, et l'aluminium, ainsi que des moules pour les pièces du jeu de construction en plastique LEGO $^{\circledR}$.

\section{CARACTÉRISATION DES SURFACES PAR BOMBARDEMENT IONIQUE}

\subsection{La rétrodiffusion Rutherford}

L'étude par Rutherford au début du siècle de la rétrodiffusion des particules $\alpha$ par une feuille mince d'or a donné suite au modèle nucléaire de l'atome, mais ce n'était que depuis les années septante que la possibilité d'utiliser un faisceau d'ions est exploitée pour l'analyse des surfaces.

Dans cette technique, appellée spectrométrie HEIS (pour « High-Energy Ion Scattering 》) ou plus communément aujourd'hui RBS (pour « Rutherford Backscattering Spectrometry »), un ion léger d'une énergie de quelques $\mathrm{MeV}$ est incident sur la surface à être sondée. Les ions pénètrent dans le solide, et commencent aussitôt à être ralentis par des interactions inélastiques avec les électrons de la matrice. Il n'y a pas de dommage à la structure à ce stade-ci (ni d'ailleurs de pulvérisation cathodique à la surface). À un certain point l'ion frappe un noyau de la matrice, et subit une diffusion élastique. Après un choc quasifrontal (généralement coulombien), l'ion revient vers la surface avec une énergie qui dépend des masses du projectile et de la cible, ainsi que de l'angle de la diffusion. L'ion est ralenti de nouveau par des électrons avant d'atteindre la surface. Le détecteur capture l'ion qui a quitté la surface, créant une impulsion dont l'amplitude est proportionnelle à l'énergie de l'ion détecté. Cette énergie (pour un angle de diffusion donné) dépend donc de la masse et de la profondeur de l'atome-cible frappé. La section efficace pour la rétrodiffusion (généralement mesurée à un angle de $170^{\circ}$ par rapport au faisceau incident) est tellement petite que la probabilité que l'ion ait subi plus d'une collision est très faible. Un spectre en énergie des ions rétrodiffusés donnera ainsi une image de la distribution en masse et en profondeur des atomes de l'échantillon étudié.

La figure 7 présente un spectre RBS typique. (L'abscisse représente l'énergie de l'ion rétrodiffusé.) Nous avons déposé cinque paires de couches (10 nm Au / $30 \mathrm{~nm} \mathrm{Ni)} \mathrm{sur} \mathrm{un} \mathrm{substrat} \mathrm{de} \mathrm{silicium.} \mathrm{Un}$ faisceau d'ions d' $\mathrm{He}^{+}$à $2,8 \mathrm{MeV}$ sonde la surface. Le détecteur est à $170^{\circ}$ par rapport au faisceau incident. (Contrairement à ce qui peut être déduit du dessin, la surface de l'échantillon était inclinée à un angle de $22^{\circ}$ par rapport au faisceau. Avec cette géométrie en incidence quasi-rasante, le faisceau parcourt une plus grande distance dans la cible, et l'échelle d'énergie perdue est étalée sur une plus grande gamme, ainsi améliorant la résolution en profondeur.)

La section efficace de diffusion coulombienne $\sigma$ est proportionnelle au carré de la charge nucléaire $\mathrm{Z}_{2}$ de l'atome-cible, d'où l'amplitude plus grande pour les signaux provenant de l'or que du nickel ou du silicium. Puisque $\sigma$ est inversement proportionnelle au carré de l'énergie de la collisión, il y a croissance du signal du silicium provenant des atomes plus profonds, et donc des ions de plus faible énergie (vers la gauche du spectre). Pour ce qui est de la sensibilité de la technique, on peut citer un exemple récent dans notre laboratoire d'une cible de césium (moins d'une monocouche) enfouie dans une interface $\mathrm{Si} / \mathrm{SiO}_{2}$ que nous avons pu détecter sans effort particulier. Il est évident que la RBS est moins sensible pour les atomes légers, et carrément impossible pour l'hydrogène et l'hélium. La sensibilité peut être augmentée dans certains cas par l'utilisation d'un faisceau d'ions plus lourds (par exemple le lithium [52], le carbone [53] ou le néon [54]), mais à ce moment-là, la profondeur ultime qu'on peut sonder est réduite. De plus, la résolution en énergie est moins bonne due au détecteur. Une résolution en profondeur de quelques dizaines de nm est typique en RBS. Cette technique fournit la concentration absolue en profondeur, et ce, de plusieurs éléments simultanément. Par contre, elle ne donne aucune information sur les liens chimiques. 
Il est intéressant de noter que la RBS peut aussi donner des bons résultats à des énergies plus basses (environ $100 \mathrm{keV}$ ) [55]. Dans ces conditions, il faut ajouter un analyseur d'énergie (généralement électrostatique). Une résolution en profondeur de quelques $\AA$ est possible, ainsi qu'une très grande sensibilité (on se rappelle que $\sigma$ varie comme $\mathrm{E}^{-2}$ ). Par contre, à ces basses énergies, une partie des ions sont neutralisés, n'arrivant pas au détecteur, et les mesures de concentration ne sont plus absolues. Pour les cristaux, on peut aussi détecter le niveau de désordre en surface.

\subsection{La détection des ions de recul élastique}

Il est évident pour des raisons de cinématique qu'un faisceau d'hélium ne peut pas être rétrodiffusé par des noyaux ni d'hélium ni d'hydrogène. (Un faisceau de protons risque d'induire des réactions nucléaires à ces énergies, rendant ainsi l'analyse des spectres plus difficile.) De plus, détecter des traces d'un élément léger parmi des atomes lourds d'une matrice devient très difficile car les (faibles) signaux provenant des atomes légers sont superposés sur un fond très élevé dû aux autres atomes lourds de la matrice. Or, souvent on a besoin de détecter la présence ou le mouvement des atomes légers dans une matrice de grande masse atomique (par exemple, dans l'étude du stockage de l'hydrogène dans les métaux).

Pour palier à ces difficultés, L'Écuyer et al. [56] ont inversé la géométrie de la RBS en inventant la technique d'analyse par détection des ions de recul élastique, appelée ERDA (pour « Elastic Recoil Detection Analysis »). Dans cette nouvelle géométrie, un faisceau d'ions lourds est incident à un angle rasant (typiquement $15^{\circ}$ ) par rapport à la surface. Comme dans la RBS, il peut y avoir des simples collisions élastiques à plusieurs profondeurs. Mais cette fois-ci, il y a un très grand flux de particules vers l'avant (typiquement, le détecteur est placé à un angle de $30^{\circ}$ par rapport au faisceau), les ions projectiles diffusés, les atomes lourds (reculant) de la matrice et aussi les atomes légers qu'on veut détecter. Le signal provenant de ces derniers est extrêmement faible par rapport à celui généré par tous les atomes lourds s'envolant vers le détecteur. L'astuce de cette technique est qu'en plaçant une feuille mince (typiquement quelques $\mu \mathrm{m}$ de mylar ou d'aluminium), les ions lourds peuvent être complètement arrêtés dans la feuille, alors que les ions légers qu'on veut détecter traverseront la feuille, et seront détectés après avoir été ralentis (mais pas arrêtés) par la feuille. Ceci est dî au fait que le freinage d'un ion dans un solide est plus marqué pour un atome lourd que pour un atome léger.

La technique peut être raffinée considérablement par le remplacement de la feuille par un spectromètre de temps-de-vol, ce qui augmente considérablement la résolution [57]. D’autres améliorations, telles que la détection en coïncidence et l'utilisation de télescopes $\mathrm{E}-\Delta \mathrm{E}$, sont résumées dans une revue récente sur le sujet [58].

\section{CONCLUSIONS}

Les faisceaux d'ions énergétiques peuvent servir à modifier ou à caractériser les matériaux avancés. La possibilité de former des composés et des structures loin de l'équilibre thermodynamique ouvre la voie à des matériaux qui possèdent des propriétés irréalisables par d'autres techniques. Les mêmes faisceaux peuvent aussi servir à déterminer de façon absolue et non-destructive la composition en profondeur des surfaces. 


\section{Références}

[1] Hirvonen, J.K., «Ion implantation » (Academic Press, N.Y., 1980).

[2] Townsend, P. D. « Uses of Ion Implantation » Materials and Processes for Surface and Interface Engineering, Y. Pauleau,

Ed. (Kluwer, Dodrecht, 1995) p. 285-306.

[3] Agius, B, et al., "Surfaces, interfaces et films minces " (Bordas, Paris, 1990) Chapitre 4.

[4] Pranevicius, L. « Coating Technology: Jon Beam Deposition » (Satas \& Associates, Warwick, R.I., USA, 1993).

[5] Hartley, N.E.W., Swindlehurst, W.E., Dearnaley, G., et Turner, J. F., J.;Mater. Sci. 8 (1973) 900-904.

[6] Hirvonen, J.K., « Ion Beam Assisted Thin Film Deposition » Materials and Processes for Surface and Interface

Engineering, Y. Pauleau, Ed. (Kluwer, Dodrecht, 1995) p. 307-346.

[7] Nastasi, M. et Mayer, J.W., Mater. Sci. and Eng. R12 (1994) 1-52.

[8] Kelly, R., Miotello, A., Knystautas, É.J. et Lo Russo, S., Surface and Coatings Technology 83 (1996) $156-161$.

[9] Bolse, W., Weber, T. et Lohmann, W., Nucl. Instr. and Methods BS0 (1990) 416-419.

[10] Knystautas, É. J., Lo Russo, S., Kelly, R., et Miotello, A., J. Appl. Phys. 80 (1996) 2702-2711.

[11] Sioshansi, P. et Smidt, F.A. « Ion implantation as a surface treatment to modify friction and wear of materials »

Engineered Materials for Advanced Friction and Wear Applications - an International Conference, Gaithersburg, Md., USA, du 1 au 3 mars 1988, F.A. Smidt et P.J. Blau, Ed. (ASM International, 1988)

[12] Tsaur, B.Y. et al., Nucl. Instr. and Methods 182/183 (1981) 67-77.

[13] Thomé, L., Benkoulal, T. et Jagielski, J., J. Appl. Phys. 75 (1994) 181-185.

[14] Rauschenbach B. et Heera, V., Defect and Diffusion Forum 57/58 (1988) 143-188.

[15] Wie, C.R.et al., Nucl. Instr. and Methods B9 (1985) 20-24; Jacobson, S., Jonson, B. et Sundquist, B. Thin Solid Films 107 (1983) 89-98.

[16] Iwaki, M., Nucl. Instr. and Methods B37/38 (1989) 661-666.

[17] Yust, C.S. et McHargue, C.J. « Microstructure and mechanical properties of ion-implanted ceramics » Emergent process methods for high technology ceramics, R.F. Davis et al., Ed. (Plenum, N.Y., 1984) p. 533-547.

[18] Wei, W. et al., Surface and Coatings Technology 37 (1989) 179-182.

[19] Pivin, J.C. Thin Solid Films 263 (1995) 185-193; Rao, G.R., Monar, K., Lee, E.H, et Treglio, J.R. Surface and Coatings Technology 64 (1994) 69-74.

[20] Lee, E.H., Rao, G.R., Lewis, M.B. et Mansur, L.K. Nucl. Instr. and Methods B74 (1991) 326-330.

[21] Bhattacharyay, A. et al. J. Phys. D29 (1996) 2745-2749.

[22] Hubler, G.K. et Smidt, F.A., Nucl. Intr. and Methods B7/8 (1985) 151-157.

[23] Baazi, T., Knystautas, É.J. et Fiset, M., Appl. Surf. Sci. 64 (1993) 133-146.

[24] Schechtman, D., Blech, I., Gratias, D. et Cahn, J.W., Phys. Rev. Lett. 53 (1984) 1951-1953.

[25] Pauling, L., Nature 317 (1985) 512-514.

[26] Kelton, K.F., Intl. Metals Review 38 (1993) 105-137.

[27] Lubensky, T.C., Ramaswamy, S. et Toner, J, Phys. Rev. B33 (1986) 7715-7719.

[28] Lilienfeld, D.A. et al., Phys. Rev..Lett. 55 (1985) 1587-1590.

[29] Larouche, B., mémoire de maîtrise, Université Laval (1989).

[30] Villars, P., Phillips, J.C. et Chen, H.S., Phys. Rev. Lett. 57 (1986) 3085-3088.

[31] Tartas, J. et Knystautas, É. J., J. Mater. Res. 6 (1991) 1219-1229.

[32] Tartas, J. et Knystautas, É. J., J. Mater. Sci. 29 (1993) 6011-6018.

[33] Townsend, P.D., Chandler, P.J. et Zhang, L. « Optical Effects of Ion Implantation » (Cambridge Univ, Press, 1994)

[34] Kulish, J.R. et al., J. Appl. Phys. 63 (1988) 2517-2521.

[35] Frank, W.F.X. et al., "Optical waveguides in polymer materials by ion implantation », Photopolymer Device Physics, Chemistry and Applications II, (vol. 1559) R.A. Lessard, Ed. (SPIE - Intemational Society for Optical Engineering, 1991) p. 344-353.

[36] Ghailane, F. et al., J. Opt. Soc. Amer. 12 (1995) 1683-1686.

[37] Chandler, P.J. et Lama, F.L., Optica Acta 33 (1986) 127-143.

[38] Mazzoldi, P., Nucl. Instr. and Methods 209/210 (1983) 1089-1098.

[39] Beauvais, J. et al., Thin Solid Films 182 (1989) 47-52.

[40] Beauvais, J. et al., Appl. Phys. A51 (1990) 515-519; Appl. Phys. A53 (1991) 249-254.

[41] Beauvais, J. et al., Appl. Phys. Lett. 57 (1990) 1354-1356.

[42] Mazzoldi, P., Nucl. Instr. and Methods B91 (1994) 478-492.

[43] Gonella, F. et al., Nucl. Instr. and Methods B127 (1997) 562-565.

[44] Strafford, K.N., Surface and Coatings Technology 81 (1996) 106-117.

[45] Garside, B.L., Mater. Sci. and Eng. A139 (1991) 207-213.

[46] Conrad, J.R. et al., J. Appl. Phys. 62 (1987) 4591-4596.

[47] Wei, R. et al., Surface and Coatings Technology 83 (1996) 235-242. 
[48] Kohlhof, K., Nucl. Instr. and Methods B106 (1995) 662-669.

[49] Müller, H.R. et Ensinger, W.J., J. of Metals (April 1995) 20-25.

[50] Pons, M., Galerie, A. et Caillet, M. J. Mater. Sci. 21 (1986) 2697-2704.

[51] Strade, C.A. Nucl. Instr. and Methods B113 (1996) 161-166.

[52] Norbeck, E., Li, L.W., Lin, H.H. et Anderson, M.E., Nucl. Instr, and Methods B9 (1985) 197-200.

[53] Leavitt, J.A., Nucl. Instr, and Methods B24/25 (1987) 717-721.

[54] Moreau, C. et al., Nucl. Instr. and Methods 218 (1983) 111-115.

[55] Buck, T.M. et Wheatley, G.H., Surf. Sci. 33 (1972) 35-55.

[56] L'Écuyer et al., J. Appl. Phys. 47 (1976) 381-382.

[57] Groleau, R., Gujrathi, S.C. et Martin, J.P., Nucl. Instr. and Methods 218 (1983) 11-15.

[58] Bik, W.M.A. et Habraken, F.H.P.M., Rep. Prog. Phys. 56 (1993) 859-902. 